\title{
Theory and simulations of squeeze-out dynamics in boundary lubrication
}

\author{
S. Zilberman \\ School of Chemistry, Tel Aviv University, Tel-Aviv, Israel 69978
}

B. N. J. Persson

IFF, FZ-Jülich, D-52425 Jülich, Germany

A. Nitzan

School of Chemistry, Tel Aviv University, Tel-Aviv, Israel 69978

(Received 20 June 2001; accepted 3 October 2001)

\begin{abstract}
The dynamics of expulsion of the last liquidlike monolayer of molecules confined between two surfaces (measured recently for the first time [J. Chem. Phys. 114, 1831 (2001)]) has been analyzed by solving the two-dimensional Navier-Stokes equation combined with kinetic Monte Carlo simulations. Instabilities in the boundary line of the expelled film produce a rough boundary for all length scales above a critical value. The squeeze-out of liquid is shown to result from the 2D-pressure gradient in the lubrication film in the contact area. The Monte Carlo simulations agrees well with experiments, reproducing most qualitative and quantitative features. In particular it shows the formation of small islands, which (in the absence of pinning mechanism) drift slowly to the periphery of the contact area. We calculate the drift velocity analytically as a function of the distance of the island to the periphery of the contact area. Experiments indicate that some kind of pinning mechanism prevails, trapping fluid pockets for very long times. When including such pinning areas in the simulations, three distinct squeeze phases and time scales were observed: (1) initial fast squeeze of most of the fluid; (2) slower squeeze of unpinned fluid pockets; (3) long term pinning of fluid pockets. We also show that a distribution of small pinning areas may produce a synergistic effect, slowing down the second phase of the squeeze, compared to a small number of big pinning areas. The paper presents a new stochastic numerical approach to problems of moving boundaries which naturally accounts for thermal fluctuations and their effect in unstable dynamics. (C) 2001 American Institute of Physics. [DOI: 10.1063/1.1421105]
\end{abstract}

\section{INTRODUCTION}

Sliding friction is one of the oldest problems in physics, and has undoubtedly a huge practical importance. ${ }^{1-3}$ In recent years, the ability to produce durable low-friction surfaces and lubricants has become an important factor in the miniaturization of moving components in technologically advanced devices. For such applications, the interest is focused on the stability under pressure of thin lubricant films, since the complete squeeze-out of the lubricant from an interface may give rise to cold-welded junctions, resulting in high friction and catastrophically large wear.

In this paper we investigate the late stages of the approach of two elastic solids limited by two curved surfaces, wetted by a lubricant film of microscopic thickness. ${ }^{4}$ Under these conditions, the behavior of the lubricant is mainly determined by its interaction with the solids that induce layering in the perpendicular direction. ${ }^{5-12}$ The thinning of the lubrication film occurs stepwise, by expulsion of individual layers. These layering transitions appear to be thermally activated. ${ }^{13,14}$ Under strong confinement conditions, some lubricants become solidlike. ${ }^{5-12}$ Other liquids, notably water, ${ }^{15,16}$ remain liquidlike up to the last layer that can be removed upon squeezing. The system considered here belongs to the second class.

We study the spreading of the $n=1 \rightarrow 0$ layering transition, which was recently observed for the first time, ${ }^{17-19}$ by imaging the lateral variation of the gap between the solid surfaces as a function of time. As explained below, the state $n=0$ corresponds to two strongly bound monolayer (one bound to each plate) that cannot be removed by squeezing. We present a detailed discussion of the dynamics of the boundary line separating the $n=1$ and $n=0$ regions during squeeze-out. In an earlier paper we discussed the nature of the layering transition when the lubrication film is in a $2 \mathrm{D}-$ solidlike state. ${ }^{20,21}$

The dynamics of the layering transition has been studied with the Surface Forces Apparatus by imaging the gap region in two dimensions. ${ }^{17}$ The experiment was performed with a chain alcohol $\mathrm{C}_{11} \mathrm{H}_{23} \mathrm{OH}$, where the amount of liquid expelled in the layering transitions during slow approach experiments corresponds to a bilayer of molecules with the OH-groups pointing toward each other. ${ }^{19}$ In the much faster approach used in Ref. 17 the structure of expelled liquid layer is not known, and could also be in the form of a single monolayer of flat lying molecules. This, however, does not change the nature of the phenomena. The mica surfaces are covered by strongly bound (via the OH-group) monolayers of $\mathrm{C}_{11} \mathrm{H}_{23} \mathrm{OH}$, that cannot be removed by squeezing, leading effectively to a $\mathrm{CH}_{3}$-terminated substrate for any additional material inside the gap. ${ }^{18}$ These coated surfaces are very inert, and the additional alcohol does not wet the surfaces. Shear experiments showed that the static friction force re- 


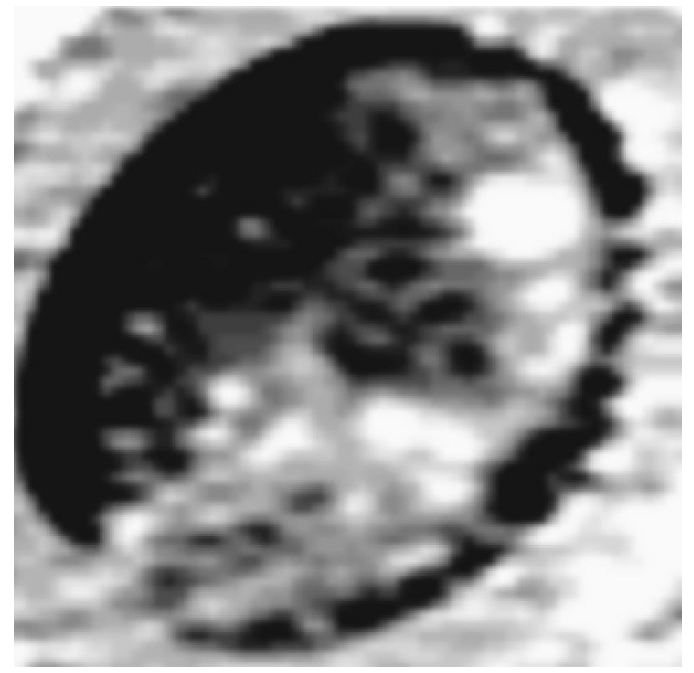

FIG. 1. Snapshot during squeeze out $n=1 \rightarrow 0$. From Ref. 17 .

mains zero (and no stick-slip is observed) up to and including the last alcohol layer, indicating that this layer $(n=1)$ is in a 2D-liquidlike state. This is further supported by viscosity measurements performed by studying the damping of mica oscillations, ${ }^{18}$ which shows liquidlike behavior down to the last expelled layer. Once this layer is expelled (corresponding to the $n=0$ situation), the contact between the $\mathrm{CH}_{3}$-terminated films leads to solidlike friction, with a nonzero static friction force, and stick-slip during sliding. The experimental data in Ref. 17 correspond to the $n=1 \rightarrow 0$ layering transition.

The dynamics of the layering transition separates into two phases. In the first phase, the system is trapped in a metastable state at the initial film thickness, i.e., one (bi-)layer of alcohol molecules between the substrate-bound monolayer. Thermal fluctuations of the two-dimensional density in this layer eventually lead to the formation of a hole with a radius that exceeds the critical radius. Once the nucleus is formed the growth phase begins, and the rest of the layer is quickly expelled. A snapshot picture taken during squeeze-out is shown in Fig. 1 (see also Ref. 17). The circle essentially marks the contact area out of which the fluid will eventually squeeze out. The bright and dark regions correspond to remaining fluid and squeeze-out areas, respectively. The squeeze process from which this snapshot was taken has started at the upper left side of the contact area, and is seen to have propagated faster along the edges of this area. ${ }^{22}$

The paper is organized as follows. Section II presents the general theory of hydrodynamic squeeze out, and a special derivation for the dynamics of small fluid pockets. Then Sec. III discusses the details of the numerical method and simulation results are analyzed. We conclude in Sec. IV with summary and conclusions.

\section{THEORY}

In this paper we consider the dynamics of expulsion for 2D-liquidlike films. We focus on the evolution of the boundary line separating the $n=1$ and $n=0$ regions during the layering transition $n=1 \rightarrow 0$ when the nucleation of the lay- ering transition occurs off-center. Since the lubrication film is assumed to be in a 2D-liquidlike state, the basic equations of motion for the lubrication film are the continuity equation and the (generalized) Navier-Stokes equation for the 2Dvelocity field $\mathbf{v}(\mathbf{x}, t)$ (we assume an incompressible $2 \mathrm{D}$ fluid): ${ }^{1,13}$

$$
\begin{aligned}
& \nabla \cdot \mathbf{v}=0 \\
& \frac{\partial \mathbf{v}}{\partial t}+\mathbf{v} \cdot \nabla \mathbf{v}=-\frac{1}{m n_{\mathrm{a}}} \nabla p+\nu \nabla^{2} \mathbf{v}-\bar{\eta} \mathbf{v},
\end{aligned}
$$

where $p$ is the 2D-pressure, $\nu$ the 2D-kinematic viscosity, and $m n_{\mathrm{a}}$ is the mass density. The last term in Eq. (2) describes the "drag-force" from the solid walls acting on the fluid. It is possible to show (see Appendix A) by dimensional arguments that, to a good approximation, one can neglect the nonlinear and the viscosity terms in Eq. (2), and assume that the velocity field changes so slowly that the time derivative term can be neglected. Thus

$$
\nabla p+m n_{a} \bar{\eta} \mathbf{v}=0 .
$$

This quasi-static approximation assumes that the flow field is able rearrange itself much faster than the interfacial line motion. From Eq. (3) it follows that

$$
\mathbf{v}=\nabla \phi,
$$

where

$$
\phi=-p / m n_{a} \bar{\eta} .
$$

The continuity equation (1) then gives

$$
\nabla^{2} \phi=0 \text {. }
$$

Experimental results ${ }^{17,18}$ indicates that the squeeze-out process is affected by pinning centers, where fluid islands are trapped. To account for this behavior, we assume that the friction $\bar{\eta}$ [see Eq. (2) above] may be position dependent. More explicitly, we assume that most of the fluid is subjected to a "regular" friction (estimated to be of order $\bar{\eta}=10^{13} \mathrm{~s}^{-1}$, Refs. 17, 18), and much higher value at some pinning regions. Equation (6) is then replaced by

$$
\nabla \cdot[\epsilon(\mathbf{r}) \nabla p]=0
$$

which has the form of a Laplace equation with a position dependent dielectric function

$$
\epsilon(\mathbf{r})=\frac{1}{m n_{a} \eta(\mathbf{r})},
$$

where the $2 \mathrm{D}$ pressure $p$ behave analogously to an electrostatic potential. At the outer boundary $r=R$ of the contact area, the pressure $p=p_{0}$ is constant, while at the inner boundary, at the interface with the $n=0$ area, it depends linearly on the perpendicular 3D-pressure $P(r)(0<r<R)$ via the relation

$$
p_{1}(r)=p_{0}+P(r) a,
$$

where $R$ is the radius of the contact area, $p_{0}$ the spreading pressure $^{13,23}$ and $a$ the thickness of the monolayer (see Appendix B and Ref. 13). From Hertz contact theory: ${ }^{1,24}$ 


$$
P(r)=\frac{3}{2} P_{0}\left(1-\frac{r^{2}}{R^{2}}\right)^{1 / 2},
$$

where $P_{0}$ is the average perpendicular pressure (external force per unit area) on the contact area. Thus, the problem of finding $\phi$ [Eq. (6)] or $p$ [Eq. (7)] is mathematically equivalent to finding the electrostatic potential between two cylinders at different potentials, $\phi_{0}=-p_{0} / m n_{a} \bar{\eta}$ and $\phi_{1}(r)$ $=-p_{1}(r) / m n_{a} \bar{\eta}$, where the outer cylinder has a circular shape (radius $R$ ), and the inner cylinder has an unknown (time-dependent) shape that should be determined. Except for the different boundary conditions, this situation is mathematically very similar to viscous fingering, where the analogy to electrostatics has already been pointed out (see, e.g., Ref. 25). If we assume that the squeeze-out process nucleate at the center of the contact area, then the theory of Persson and Tossati, ${ }^{13}$ which assumes that the squeezed area proceeds with circular symmetry, yields the following expression for the fluid area $A(t)$ at time $t$ :

$$
\left[1-\frac{A(t)}{A_{0}}\right]\left\{\ln \left[1-\frac{A(t)}{A_{0}}\right]-1\right\}=-\frac{t}{t_{0}},
$$

where $A_{0}=\pi R^{2}$ is the contact area, and $t_{0}$ is the time it takes to completely squeeze out the fluid from the contact area. The squeeze-out time $t_{0}$ is given by ${ }^{13}$

$$
t_{0}=R / v_{0}
$$

with

$$
v_{0}=\frac{3}{2} \frac{a P_{0}}{n_{a} R m \bar{\eta}} .
$$

It is easy to show that the time evolution of the boundary line is unstable with respect to small perturbations. Consider the evolution of a circular squeeze-out region at the center of the contact area. Assume that, due to a fluctuation, a small protrusion is formed on the boundary line, which will locally decrease the distance to the outer boundary line $r=R$. By analogy to electrostatics, this will give rise to an enhanced "draining" velocity of the fluid at the protrusion, so that the boundary line at the protrusion will move faster toward the periphery than in the other regions. This argument is valid for protrusions of any size, and it follows that, within the model discussed above, the boundary line will be rough at all length scales. This argument, however, disregards the line energy. Taking into account the line tension $\Gamma$ (free energy per unit length of the boundary line), leads to a boundary line that is smooth on all length scales below some critical cutoff length $\lambda_{c}$, while it is rough on longer length scales ${ }^{14,26}$

$$
\lambda_{\mathrm{c}}=2 \pi\left(\Gamma / m n_{a} \bar{\eta} v_{0}\right)^{1 / 2},
$$

where $v_{0}$ is the velocity of the boundary line. The line tension $\Gamma$ has a contribution from unsaturated bonds at the boundary line ( $\sim 0.01 \mathrm{eV} / \AA$, Ref. 13$)$, and another much larger contribution from the energy stored in the elastic deformation field in the confining solids in the vicinity of the boundary line $(\sim 1 \mathrm{eV} / \AA$, Refs. $14,17,18)$. Under the experimental conditions of Ref. 17, Eq. (14) predicts $\lambda_{\mathrm{c}} \sim 5$ $\mu \mathrm{m}$, which equals $1 / 10$ of the diameter of the contact area.
The experimental boundary line for $\mathrm{C}_{11} \mathrm{H}_{23} \mathrm{OH}$ is indeed rough at this length scale, while it is smooth on shorter length scales. Based on this result one may also argue that the linear size of the trapped fluid islands should be of order $\lambda_{\mathrm{c}}$ (or larger), which again agrees with the observations.

Finally, let us consider the motion of a small (compared to the size of the contact area) island, located a distance $r$ from the center of the contact area. We assume the island does not experience any pinning forces. We will calculate the dependence of the radial velocity $v(r)$ of the island on its position $r$. First, note that the elastic energy stored in the deformation field in the solids in the vicinity of the island is $U \approx \pi b^{2} P(r) a$, where $b$ is the radius of the island, $r$ is the distance of the center of the island from the center of the contact area, and $a$ is the thickness of the monolayer. The radial force acting on the island is thus

$$
F=-\frac{\partial U}{\partial r}=-\pi b^{2} P^{\prime}(r) a .
$$

This force moves the island in the radial direction toward the periphery of the contact area. We assume overdamped motion, and can thus neglect the inertial force acting on the island, so that the driving force $F(r)$ must just balance the frictional drag force from the solid walls:

$$
\pi b^{2} n_{a} m \bar{\eta} v(r)=F
$$

or

$$
v=-\frac{a}{n_{a} m \bar{\eta}} P^{\prime}(r) .
$$

If $P(r)$ is given by the Hertz expression (10) then this gives

$$
\frac{v}{v_{0}}=\frac{r}{R}\left(1-\frac{r^{2}}{R^{2}}\right)^{-1 / 2},
$$

where $v_{0}$ was defined in Eq. (13). The function Eq. (18) is shown in Fig. 2(a). Note that the velocity goes to infinity for $r=R$. However, for any finite size island (radius $b$ ), when $r=R-b$ the island will make contact with the region outside the contact area; computer simulations (see Sec. III) and experiment have shown that this result in the formation of a neck toward the outside, through which the fluid will be squeezed. Using $v=d r / d t$ and $x \equiv r / R$, it is easy to integrate Eq. (18) to get the radial position $r(t)$ of the island as a function of time. In particular, the time $t=t(r)$ it takes to squeeze-out an island that starts at position $r$, is given by

$$
\frac{t}{t_{0}}=\int_{r / R}^{1} d x\left(\frac{1}{x^{2}}-1\right)^{1 / 2},
$$

where $r=r(0)$ is the initial distance of the island from the center of the contact area, and $t_{0}=R / v_{0}$. This function is shown in Fig. 2(b). If pinning centers occur at the interface, it will result in fluctuations in the velocity $v(r)$ of the island, and in a distribution of squeeze-out times [for identical starting distances $r(0)$ ]; the study of the motion of islands would hence give information about the nature of the pinning dynamics. 

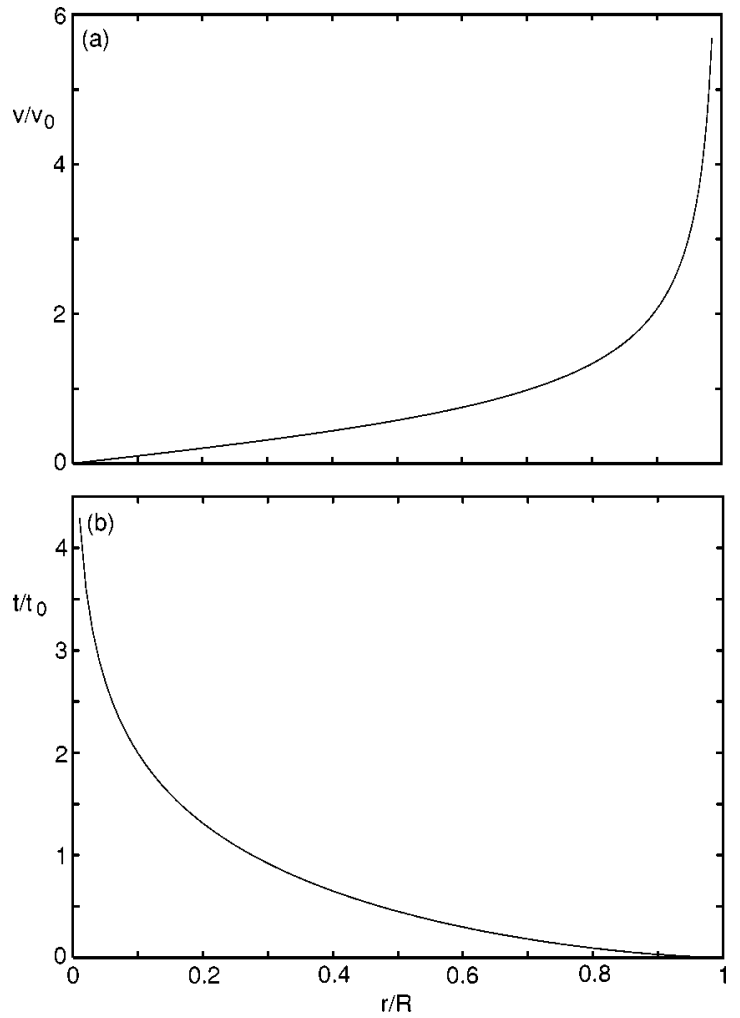

FIG. 2. (a) The radial velocity $v$ of a small island as a function of the distance $r$ from the center of the contact area. (b) The time $t$ it takes to squeeze out a small island as a function of the initial distance $r$ from the center of the contact area.

\section{COMPUTER SIMULATIONS}

\section{A. Methodology}

We have performed kinetic Monte Carlo (MC) simulations [based on Eqs. (1)-(10)] to study the squeeze-out process in detail. The basic reasoning behind the MC modeling of the system is quite straightforward. We start with a small initial squeezed region (usually taken to be circular) of radii greater then the critical radii for the formation of such a squeezed hole. The initial formation process is a separate issue that is not studied here. At each step we solve the Laplace equation on a two-dimensional cell centered grid, and move the interface line between fluid and squeezed areas in a manner that follows flow lines. In this scheme we impose continuity of the flow velocity across cell boundaries, which is equivalent to the continuity of the dielectric displacement in electrostatics.

We have used an area discretization scheme ${ }^{27}$ combined with a successive over relaxation iterative procedure for solving the Laplace equations (6), (7) ${ }^{28}$ We used a square grid, typically of size $280 \times 280$. This grid divides the system into square cells and the grid points are taken to be at their centers. The interfacial line is taken along these grid lines, so that a grid cell has a unique attribute, either squeezed or fluid. As discussed in Sec. II, the boundary conditions are $p=p_{0}$ on the external periphery $(r=R)$, and $p=p_{1}(r)$ [cf. Eq. (9)] on the interfacial line between squeezed and fluid areas at position $r$. The actual value of the spreading pressure $p_{0}$ is unimportant, and it was set to zero in our calculations.
Numerical solutions of moving boundaries problems could in principle be obtained using deterministic boundary evolution algorithms (e.g., Ref. 29) which generates deterministic solutions within the numerical accuracy. As discussed in Sec. II and in Ref. 14, the natural instability of the Laplace flow model Eqs. (6), (7) makes the time evolution of the interfacial lines sensitive to random thermal fluctuations. Therefore, we have chosen to use a stochastic line propagation mechanism, which on the average propagates the interface in the flow directions on the one hand, and naturally incorporates fluctuations on the other. The mechanism discussed below has the advantage of simplicity, compared to deterministic numerical line propagators.

Having solved the Laplace equations (6), (7) we get the pressure field $p$ from Eq. (5) on every grid point. Next we associate a velocity with every segment of the interfacial line separating a fluid and a squeezed cell, using the given value of potentials $\phi$ at the center of the corresponding fluid cell and the interfacial line. The latter is given from the boundary condition, Eq. (9). By definition, this velocity is perpendicular to the corresponding line segment. These velocities are used to determine the propagation of the interfacial line, i.e., the conversion of interfacial cells from fluid to squeezed, or from squeezed to fluid state, according to the following algorithm. Define for each such cell $I$ the velocity parameter

$$
v_{I}=\sum_{j(I)}\left|v_{j(I)}\right|,
$$

where the sum is over those boundary line segments $j(I)$ of cell $I$ for which the line velocity computed above points towards the cell $I$. We use the resulting $v_{I}$ as a measure of the tendency of this cell to switch its state. The probability that a switch takes place in the current Monte Carlo step is taken to be

$$
P_{V}(I)=\frac{v_{I}}{\max \left\{v_{I}\right\}} .
$$

Thus the probability to accept a tentative MC move that changes the state of cell $I$ is linearly proportional to $v_{I}$, meaning that on average the interface line would change as determined by the velocity field. Note, however, that the physical time is not simply proportional to the number of MC steps because the velocity normalization in Eq. (20) is different at each such step. The physical time increment associated with a single MC step should therefore be counted as $\Delta t \propto 1 / \max \left\{v_{I}\right\}$. We define the MC time of the process by

$$
\tau_{\mathrm{MC}}=\sum_{\text {steps }} \frac{1}{\max \left\{v_{I}\right\}} .
$$

With this definition, the real physical time is proportional to the MC time

$$
t=\alpha \tau_{\mathrm{MC}} .
$$

A possible way to determine $\alpha$ is discussed below.

At each MC step the interfacial propagation should be supplemented by a line smoothening process that simulate the effect of the line tension. We have chosen a stochastic line relaxation process, in which neighboring squeezed and 

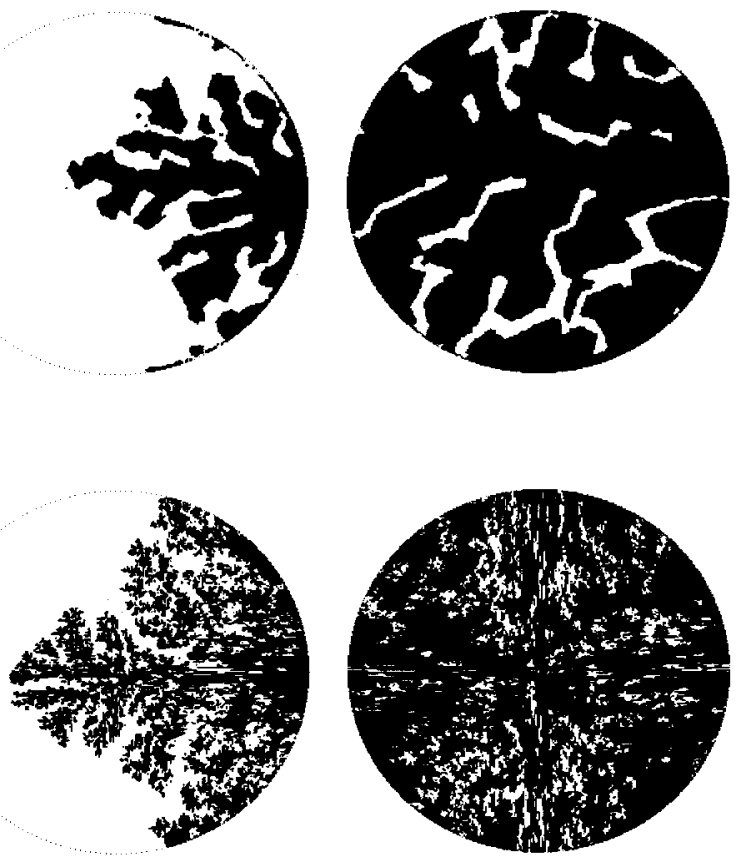

FIG. 3. Snapshots of the layering transition computed with Hertz contact pressure, with (top) and without (bottom) taking line tension into account (see text for details). The black section represent squeezed-out area, and the white section is fluid area. Time propagation is from left to right. fluid cells may interchange so as to reduce the line energy. Each line relaxation step is taken to be composed of $\mathrm{M}$ micro exchange steps, where $\mathrm{M}$ is the number of squeezed cells along the interface line. At each micro step, a squeezed interface cell and a neighboring fluid cell are chosen at random for an exchange process. The probability for accepting the exchange is

$$
P_{l}(E)= \begin{cases}q & \Delta E \leqslant 0 \\ q \mathrm{e}^{-\beta \Delta E} & \Delta E>0,\end{cases}
$$

where $q=\min \left(1, \bar{\eta}_{\mathrm{s}} / \bar{\eta}_{\mathrm{f}}\right)$, and $\bar{\eta}_{\mathrm{s}}, \bar{\eta}_{\mathrm{f}}$ are the values of the sliding friction $\bar{\eta}$ at the squeezed and fluid cells, respectively. $\Delta E=\Gamma \Delta l$ is the change in line free energy; $\Gamma$ is the line tension and $\Delta l$ is the change in the length of the interface line caused by the exchange. The $q$ factor is added to the normal Metropolis criteria in order to prevent the line relaxation steps to easily move fluid patches out of high friction areas, as discussed above.

For every MC step we usually had few tens of line relaxation steps, and unless specified otherwise, line tension of $1 \mathrm{eV} / \AA$ was used. It should be noted that the number of line relaxation moves should be restricted, in order to not change the center of mass position of small (compare to mesh size) fluid pockets. This restriction still leaves a large margin where this number does not affect the resulting dynamics.

An alternative way to introduce the line tension effect is via the boundary conditions. It has been shown ${ }^{14}$ that the line tension adds another curvature dependent term to the boundary conditions at the interface between squeezed and fluid area. Then the boundary conditions Eq. (9) changes locally to $p_{1}^{\prime}(r)$, where

$$
p_{1}^{\prime}(r)=p_{1}(r)-\kappa \Gamma,
$$

where $\kappa$ is the local curvature and $p_{1}(r)$ was defined in Eq. (9). This approach is harder to implement in a grid based method, but both treatments should give the same result if enough boundary relaxation moves are used, so that the boundary line is close to thermal equilibrium at all stages during squeeze out.

Calculation of interface length $l$ (discussed above) between squeezed and fluid areas involves some subtle issues (see Appendix C). Simply summing over the lengths of cell edges along the interface was found to be problematic. A process based on this procedure tends to form rectangularlike interfaces due to the underlying grid symmetry, unless unphysically low line tension is used. Alternatively, we calculate $l$ as the sum over straight lines connecting the centers of our grid cells. It can easily be shown that in doing so we overcome the tendency to form rectangularlike interfaces, and realistic line tension could be used in the model. However, we found very little (if any) differences between the results of the two approaches, meaning the low line energy in the first approach acts as an effective relaxation parameter.

In our calculations we have used reduced units, in which the 2D-pressure was expressed in units of the pressure $p_{c}$ at the center. The length was in units of the contact area radius $R$, and the dielectric function [Eq. (8)] was in units of its value outside the pinning areas. In all calculations, the initial squeezed area was taken to be circular with radii $0.1 R$, and unless otherwise stated, its position was centered around radial coordinate $0.7 R$.

\section{B. Numerical results}

\section{Generic behavior}

We focus on the dynamics of the layering transition $n$ $=1 \rightarrow 0$. Figure 3 (bottom) shows snapshot pictures of the layering transition for a Hertzian contact pressure and with the line tension $\Gamma=0$. The resulting boundary line is rough (fractal) on all length scales above the low distance cutoff given by the mesh size. This behavior is in sharp contrast to 

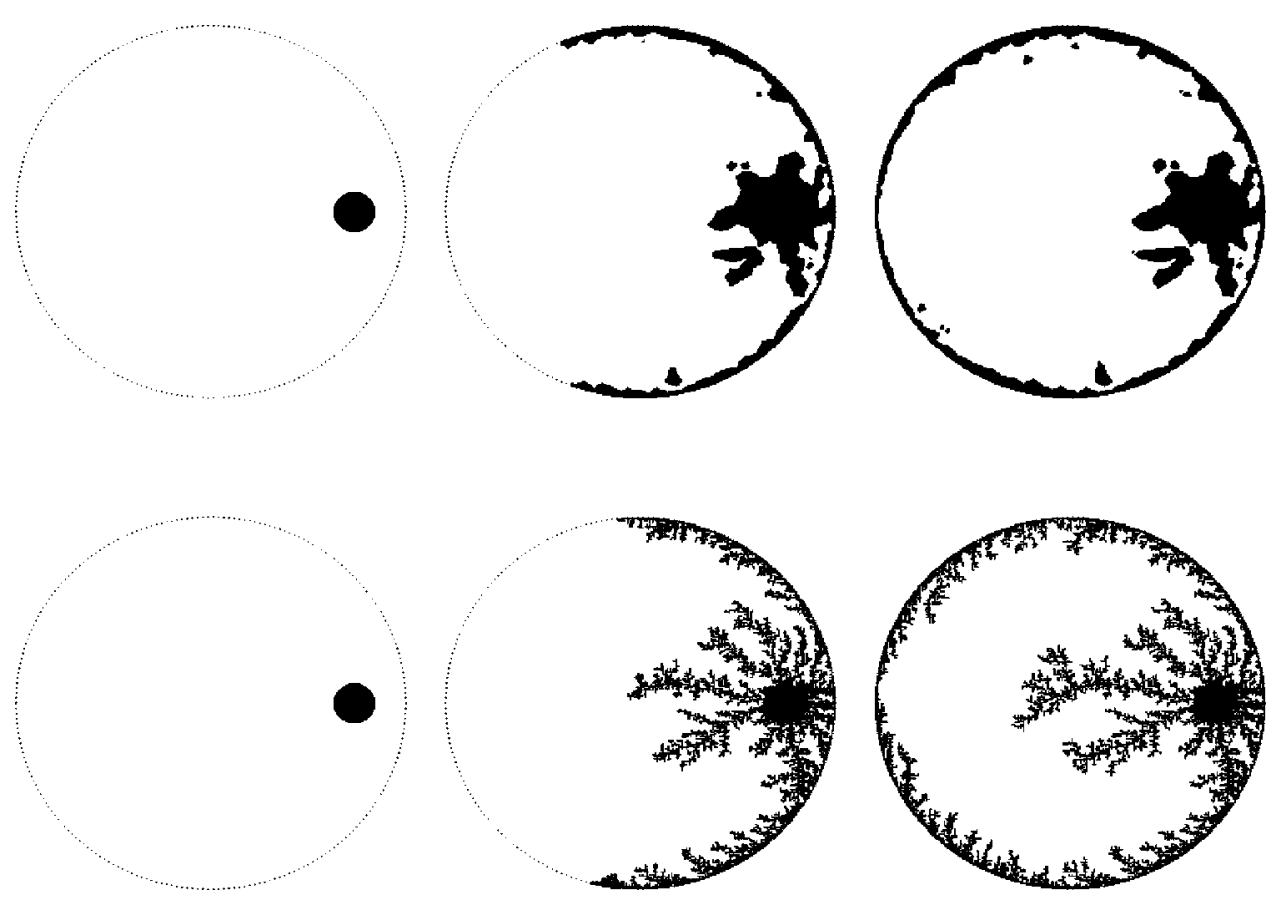

FIG. 4. Snapshots of the layering transition computed with constant contact pressure, with (top) and without (bottom) taking line tension into account (see text for details). The black section represents squeezed-out area, and the white section is fluid area. Time propagation is from left to right. experimental observations (see Fig. 1). Figure 3 (top) shows snapshots pictures of the layering transition when the line tension $\Gamma=1 \mathrm{eV} / \AA$. In this case the fractal pattern occurs on all length scales above a cutoff length $\lambda_{c}$, determined by the line tension. This illustrate the fundamental importance of the line tension for a correct description of the squeeze-out process.

Figure 4 shows the same as Fig. 3, but now with a constant contact pressure difference, corresponding to a constant perpendicular 3D-pressure. Note that the fast propagation of the boundary line along the periphery of the contact area causes trapping of a huge fluid island. When a Hertzian contact pressure is assumed, interfacial velocity close to the periphery is much smaller (since $P \rightarrow 0$ as $r \rightarrow R$ ), which makes it possible to squeeze out much more fluid from the interior of the contact area, resulting in much smaller "trapped" islands, in qualitative agreement with experiment. We also note that in Fig. 4 the squeeze-out process stops when the drained area encircles the trapped island. At this point there is no pressure difference across the fluid and the dynamics stops. This is in sharp contrast to the Hertzian contact pressure case, where a squeeze-out force acts radially on any island of "trapped" fluid. Thus, in the latter case it is necessary to introduce pinning centers in order for fluid to remain trapped for large times, as observed in the experiments.

During squeeze-out [Fig. 3 (top)] the local curvature of the boundary line between the $n=1$ and $n=0$ regions becomes negative in some areas. Some of these areas eventually detach from the boundary and leave behind pockets of $n=1$ layer trapped material in the final $n=0$ state. We note that unless an island is centered in the center of the contact area, there will be a net tangential force acting on the island due to the spatial variation in the normal stress from a maximum at the center to zero at the periphery of the contact area. Thus, without defects (pinning centers), the pockets move towards the edge as a whole. There they form little necks through which liquid is squeezed out. This is exactly what is observed in the experiments, see Fig. 1 and Ref. 17. However, in these experiments some islands are also found to be pinned, indicating that there may be defects or contamination on the solid walls used in the experiments. In order to simulate pinning we have introduced small circular (high friction) areas, where $\bar{\eta}$ was taken to be up to $10^{4}$ times higher than in the remaining area. This produces pinning of the fluid in these areas, resulting in a finite amount of trapped liquid even for very large times.

Figure 5 shows the time evolution of the relative remaining fluid area. The circles were obtained ${ }^{4}$ from the experimental results of Mugele and Salmeron, ${ }^{17,18}$ by image analysis of a squeeze sequence of snapshots such as the one shown in Fig. 1. The solid line shows the computed fractional area occupied by the fluid as a function of time for an initial position of the nucleus at $r=0.7 R$, with a concentration of pinning areas covering about $13 \%$ of the total contact area. The factor $\alpha$ relating the MC time to the real time [see Eq. (23)] has been used as a timescale fitting parameter.

\section{Time scales}

Figure 6 shows the variation of the fractional area occupied by the fluid as a function of time without (solid line) and with (dotted and dashed-dotted lines) pinning centers, assuming that the initial $n=0$ nucleus occur at $r=0.7 R$. With the pinning centers included, about $13 \%$ of the liquid remain trapped at the interface for large times.

One can clearly observe three distinct time scales associated with the squeeze-out process. The first stage is a fast squeeze-out of most of the fluid, but leaving behind many relatively big fluid pockets. The second slower stage is the squeeze-out of nonpinned fluid pockets while the third stage is the long term pinning. The second stage process takes a 


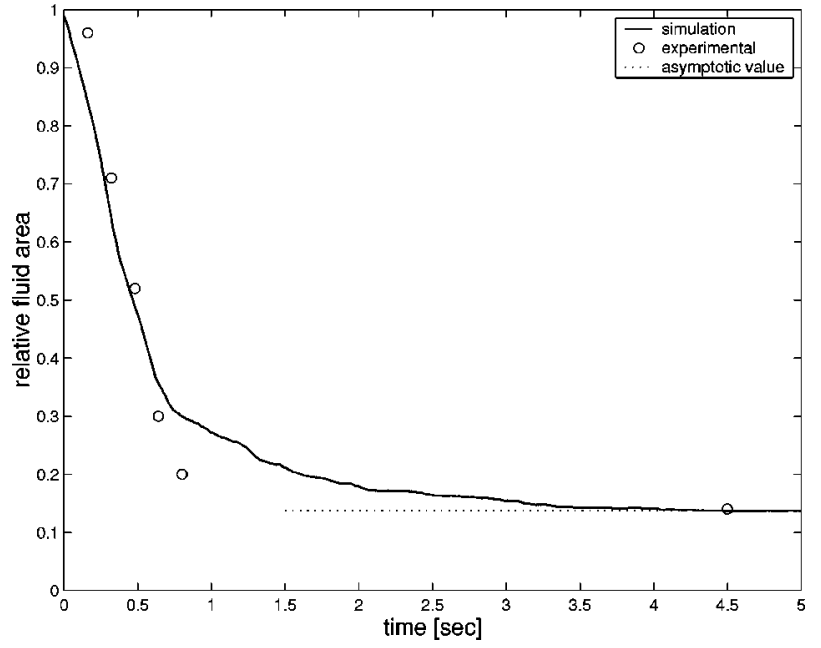

FIG. 5. The fractional area occupied by the fluid as a function of time with the initial position of the squeeze nucleus at $r=0.7 R$. Solid line: theory. Circles: experimental.

long time because islands originally located close to the center of the contact area experience a very weak net lateral force, as discussed in Sec. II.

It is easy to understand the dynamics of the first stage based on the Persson-Tosatti model [Eq. (11)]. Simulation results show that the lhs of Eq. (11) depends linearly on the MC time for short times, even for off center squeeze nucleus. Thus the model appears to qualitatively describe the very short time dynamics, however it fails at longer times (still within the first stage). The success of Eq. (11) at short time can serve as a rough way for direct time scaling of simulation results to real systems, without any fitting parameters. Equation (11) could be recasted as

$$
\left[1-\frac{A(t)}{A_{0}}\right]\left\{\ln \left[1-\frac{A(t)}{A_{0}}\right]-1\right\}=-\frac{\alpha}{t_{0}} \tau_{\mathrm{MC}},
$$

where $t_{0}$ [cf. Eq. (12)] is determined by the parameters of the physical system. Therefore using Eq. (26) in the early parts of the simulation provides an estimate of the proportionality factor $\alpha$ of Eq. (23).

During the second stage of squeeze-out, we found that the fluid area scales as $A(t) \sim t^{-b}$. When pinning centers are absent, the exponent is $b \approx 1.5$. Inclusion of pinning centers slowed down the dynamics, yielding $b \approx 0.6-0.7$ in the examples shown in Fig. 6. This indicates that the pinned areas influence the squeeze-out well beyond their physical location.

The third stage is clearly influenced by the pinning strength; increasing the relative friction in the pinning centers extends the life span of trapped fluid pockets, as expected. Therefore one can, in principle, gain information about the pinning strength of real surface defects by comparing with simulations.

\section{Other effects}

Figure 7 shows the fractional area occupied by the fluid as a function of time for an initial position of the nucleus: at the center (solid line), at $r=0.4 R$ (dotted line), and at $r$

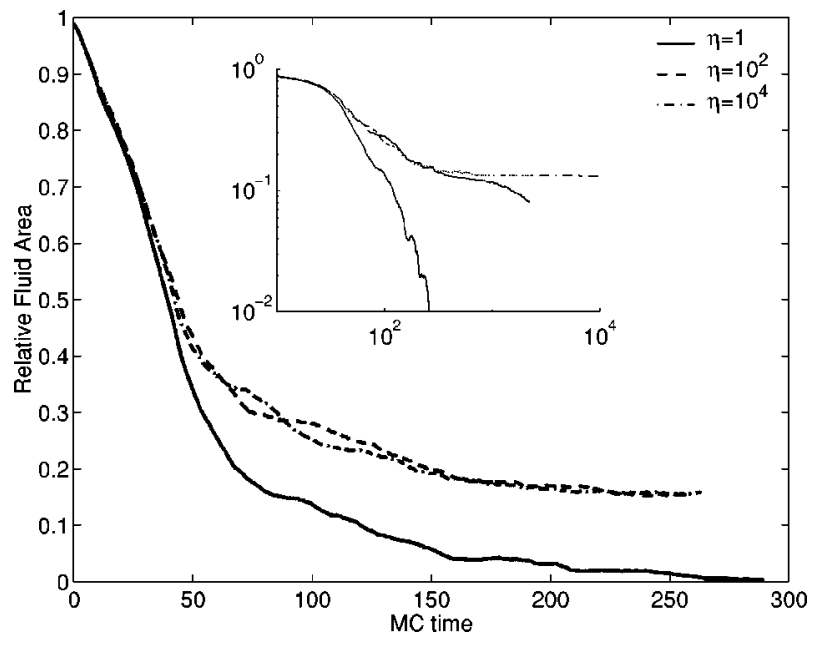

FIG. 6. The fractional area occupied by the fluid as a function of time without (solid line) and with (dashed line and dashed-dotted line) pinning centers, and with the squeeze-out nucleus at $r=0.7 R$. The inset shows the long time behavior on a $\log -\log$ scale.

$=0.7 R$ (dashed-dotted line). Note again that one can distinguish between two phases of squeeze-out: an initial "fast" phase, and a slower phase where the fluid islands are squeezed out. In contrast with the second squeeze stage, the closer the initial position to the center of the contact area, the faster is the first stage. The dynamics of the boundary line in the first stage is determined by the pressure gradient at the boundary Eq. (4) which results from the solution of Laplace equations (6), (7) with boundary conditions derived from the Hertz pressure Eqs. (9), (10). These gradients are greater the closer the interfacial line to the center. In the second stage the gradients originates mainly from the derivative of the Hertz pressure itself Eq. (10), thus becoming higher as we depart from the center (see Fig. 2).

We have also considered possible cooperative effects: would a distribution of small pinning areas be more efficient then a few big areas, in delaying the squeeze-out process.

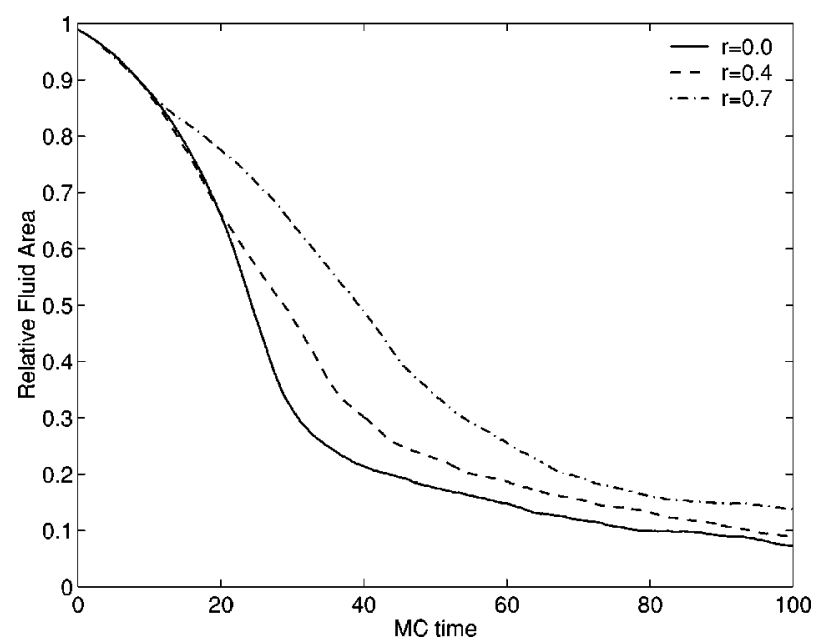

FIG. 7. The fractional area occupied by the fluid as a function of time with the initial position of the squeeze nucleus at the center (solid line), at $r$ $=0.4$ (dotted line) and for $r=0.7 R$ (dashed-dotted line). Without pinning areas. 


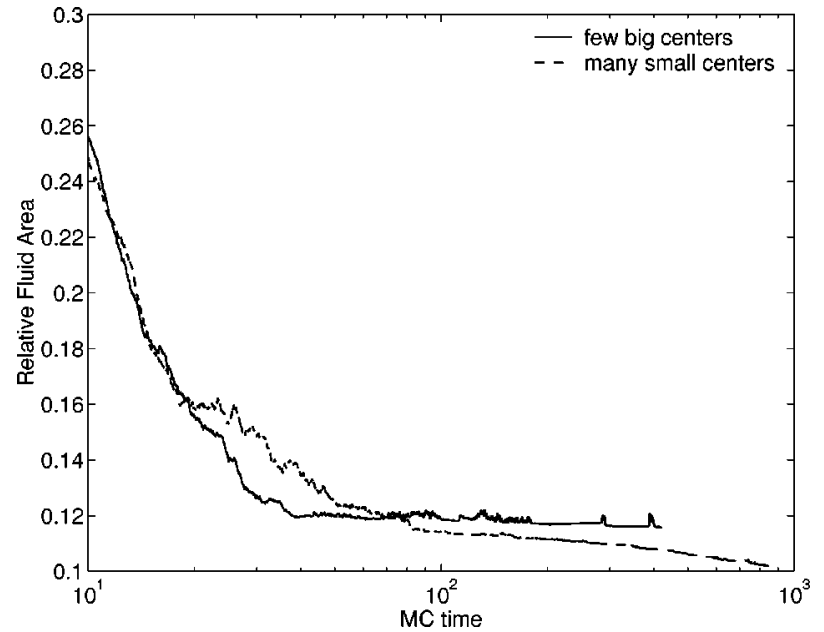

FIG. 8. Cooperative effects at the second and third phases of the squeezeout process.

Figure 8 shows two such limiting cases in which the overall high friction areas was roughly equal, about $\sim 12 \%$. The example shows that some cooperative effects may occur mainly at the late stages of the second squeeze phase. These effects were also observed in visual inspection of simulation snapshots. At the third phase the ability of small pinning centers to trap fluid for long time is weaker compared to the case of few big centers. It seems that small fluid pockets formed by small pinning areas are more sensitive to random fluctuations in which small fluid patches detach from the fluid pocket. Once it happened, they slip out due to the lateral force, as discussed in Sec. II above. We found that the cooperative effects are very sensitive to the distribution of the pinning centers; some distributions do not produce these effects at all, though the behavior at the third phase is consistent.

\section{SUMMARY AND CONCLUSIONS}

A lubrication fluid confined between two approaching surfaces form, in the limit of thin interfaces, well-defined layers of molecular thickness, whose number decreases in discontinuous steps with increasing applied pressure. We have studied the dynamics of the squeeze-out by solving the 2D Navier-Stokes equations with an interfacial friction term, and found the results to be in good agreement with the experimental data. We note that it is very important to use a variable (Hertzian) contact pressure profile and to include the line tension: If $\Gamma=0$ or if the contact pressure is assumed to be constant rather than Hertzian, the computer simulations disagree qualitatively with the experiment. We have also studied the motion of small islands, and pointed out that fluctuations in the velocity may give information about the nature of the pinning dynamics.

\section{ACKNOWLEDGMENTS}

The authors thank E. Brener and P. Graf for useful discussions. B.P. and A.N. thank BMBF for a grant related to the German-Israeli Project Cooperation "Novel Tribiological Strategies from the Nano- to Meso-Scales." B.P. thanks
Pirelli for a grant related to "Physical principles of rubber friction and application to tires." A.N. thanks the Israel Science Foundation for partial support of this work (Grant No. 248/99).

\section{APPENDIX A: DIMENSIONAL ANALYSIS OF THE NAVIER-STOKES EQUATIONS}

Here we justify neglecting various terms in the NavierStokes equations (2). In the experimental system of Mugele and Salmeron ${ }^{17,18}$ the typical length scale is $R \sim 10 \mu \mathrm{m}$ (contact area radius) and the typical squeeze-out time scale $\tau$ $\sim 1$ second. Then the relative magnitude of the terms in Eq. (2) are as follows:

$$
\frac{v}{\tau}: \frac{v^{2}}{R}: \frac{\Delta p}{R m n_{a}}: \frac{\nu v}{R^{2}}: \eta v .
$$

Now, if we divide every term in Eq. (A1) by $\eta v$ and put $v \sim R / \tau$ we get

$$
\frac{1}{\eta \tau}: \frac{1}{\eta \tau}: \frac{\tau \Delta p}{R^{2} m n_{a} \eta}: \frac{\nu}{\eta R^{2}}: 1 .
$$

Since $\eta \approx 10^{13} \mathrm{~s}^{-1},{ }^{17}$ the first two terms in Eq. (A2) are negligible. Typical values for the bulk kinematic viscosity of organic liquids is of the order of $\nu \approx 10^{-3} \mathrm{~m}^{2} / \mathrm{s}$, making the fourth term of the order of $10^{-6}$ and again negligible. Lastly, the third term has to come out to be of order $\sim 1$ in order for Eq. (2) to hold. Using Eq. (9) we see that $\Delta p=P_{0} a$, where $P_{0}$ is the external pressure and $a$ is the change in the separation between the confining solids due to the squeeze out (of the order of molecular diameter). Putting in the parameters from Ref. 17 the third term is indeed of order unity, as required.

\section{APPENDIX B: BOUNDARY CONDITIONS}

From thermodynamics we know that the pressure is given by

$$
p=\left(\frac{\partial F}{\partial V}\right)_{T},
$$

where $F$ is the free energy and $V$ is the volume. Its 2D equivalent is

$$
p=\left(\frac{\partial F}{\partial A}\right)_{T} \equiv \gamma,
$$

where $A$ is the area. The 2D-pressure is just the surface energy (per unit area), and we would express the boundary conditions by the surface energy change during squeeze-out. Now suppose the bounding solid walls are rigid and we form a hole. The free energy change per unit area when fluid flows into the hole is called the spreading pressure $p_{0}$. Everywhere else in the fluid film the pressure has to be constant and equal to the spreading pressure, otherwise fluid would flow from high to low pressure areas leaving empty holes behind (assuming constant density).

Pressure $p_{1}$ on the boundaries of the formed hole has to be greater then the spreading pressure in order for the 
squeeze out process to propagate. Its value is determined as follows. We make a small variation in the boundary line at given point, to convert a squeezed surface element into a fluid element. The free energy change is the boundary value $p_{1}$ [Eq. (B2)]. Its value would be the energy required to "lift" the confining solids to distance $a$ apart plus $p_{0}$, the energy change due to fluid spread. Therefore $p_{1}=p_{0}$ $+P(x, y) a$, and $P(x, y)$ is the local perpendicular 3Dpressure.

\section{APPENDIX C: CALCULATION OF INTERFACE LENGTH}

\section{Model I}

Within line tension model I, interface length was calculated as a sum over the rectangular edges of the cells (Fig. 9). Now we will show that within that model rectangularlike shape have lower line energy, then quasicurved ones. Hence high (realistic) values of $\Gamma$ (line tension) would render the interface to have straight rectangularlike shape. In order to avoid it one has to make the line tension small letting the thermal fluctuations make the interface more "curved" as seen in experiments.

Our starting point is the quasi-circular shape demonstrated in Fig. 10. The diameter of the circle is $2 R=N \Delta$, where $N$ is the number of cells along the diameter and $\Delta$ is the grid discretization. It follows that the perimeter of the circle is $L_{c}=4 N \Delta$. Circle area is approximately $(R \gg \Delta) S_{c}$ $=\pi R^{2} \approx \pi N^{2} \Delta^{2} / 4$. Suppose we have a rectangle of equal area as the circle $S_{r}=S_{c}$. Then its perimeter is $L_{r}=4 \sqrt{S_{r}}$ $=2 \sqrt{\pi} N \Delta$, and the ratio $L_{c} / L_{r}>1$. Therefore the quasicircle has a longer perimeter than the rectangle.

The mechanism described above is a purely model artifact, resulting from the underlying rectangular symmetry of the grid. When we use very low line tension, simulation results agree well with experiments and intuition. Nevertheless it is bothering to have a line tension which is $4-5$ orders of magnitudes smaller then real values.

\section{Model II}

A simple remedy, within the line relaxation paradigm, to the line tension problem is as follows. We could in principle calculate the interface length in a slightly modified form, connecting the centers of the cells via straight lines instead of summing over the edges (Fig. 11).

\section{Fluid Area}

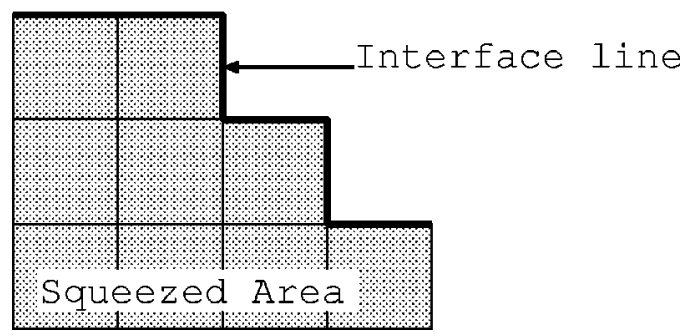

FIG. 9. Model I of interface line length between squeezed and fluid areas.

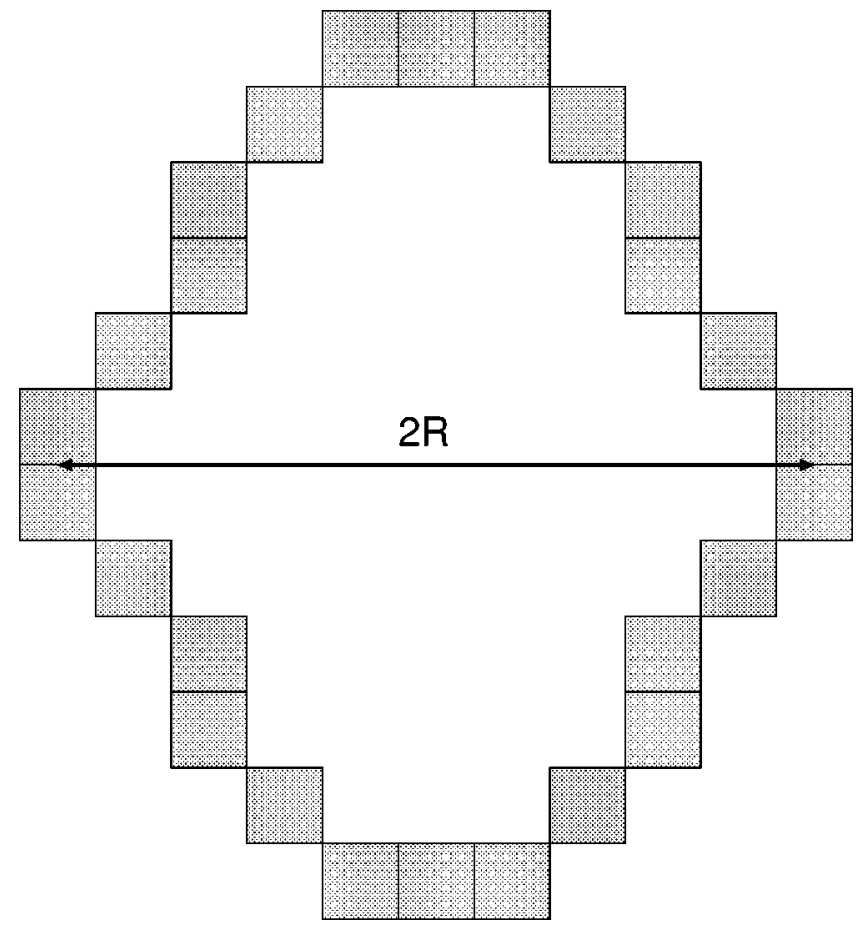

FIG. 10. Quasi-circular shape on a rectangular grid.

We start with examining one quarter of a discretized circle. Its perimeter is composed of $i$ segments parallel to the $x$-axis, $j$ segments parallel to the $y$-axis and $k$ diagonal segment. It is easy to see that in each axis direction:

$$
\begin{aligned}
& i+k=N / 2, \\
& j+k=N / 2,
\end{aligned}
$$

implying that $i=j \equiv x$ and $k=N / 2-x$. The total perimeter length of the circle is then

$$
\begin{aligned}
L_{c} & =4[2 x+(N / 2-x) \sqrt{2}] \Delta \\
& =4 N \Delta[1 / \sqrt{2}+(2-\sqrt{2}) \alpha / 2],
\end{aligned}
$$

where $\alpha=x /(N / 2)$ is the fraction of straight (nondiagonal) segments. Testing again the ratio $L_{c} / L_{r}$ we can find the maximum value of $\alpha$ for which the ratio is smaller then 1 , and it turns out to be $\alpha \approx 0.61$. Therefore even if in a given axis (say $x$-axis) we have up to $61 \%$ of straight line segments, the discretized circle would have shorter perimeter

Fluid Area

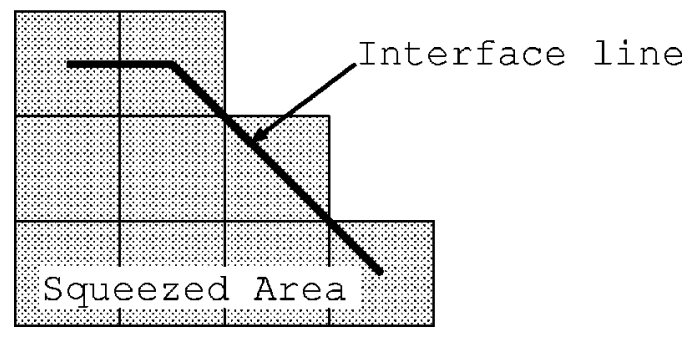

FIG. 11. Model II of the interface line length between squeezed and fluid areas. 
then the corresponding rectangle. In practice the percentage is much lower, hence favoring curved shapes naturally.

One subtle issue is how to assign consistent line energy for a squeezed area consisting of just one or two cells, resulting from a fluctuation. In reality such small fluid pockets are very unstable due to the very high line energy and small area. Since it is undesirable to have these small fluctuation, we have used an ad hoc solution in which a very high interface length is associated with them, thus preventing their formation.

${ }^{1}$ B. N. J. Persson, Sliding Friction: Physical Principles and Applications, 2nd ed. (Springer, Heidelberg, 2000).

${ }^{2}$ B. N. J. Persson, Surf. Sci. Rep. 33, 83 (1999).

${ }^{3}$ J. Krim, Sci. Am. 275, 74 (1996).

${ }^{4}$ S. Zilberman, B. N. J. Persson, A. Nitzan, E. Mugele, and M. Salmeron, Phys. Rev. E 63, 055103 (2001).

${ }^{5}$ J. N. Israelachvili, Intermolecular and Surface Forces (Academic, London, 1995)

${ }^{6}$ M. L. Gee, P. M. McGuiggan, and J. N. Israelachvili, J. Chem. Phys. 93, 1895 (1990).

${ }^{7}$ Jianping Gao, W. D. Luedtke, and Uzi Landman, Phys. Rev. Lett. 79, 705 (1997).

${ }^{8}$ H. Tamura, M. Yoshida, K. Kusakabe, C. Young-Mo, R. Miura, M. Kubo,

K. Teraishi, A. Chatterjee, and A. Miyamoto, Langmuir 15, 7816 (1999).

${ }^{9}$ L. Demirel and S. Granick, Phys. Rev. Lett. 77, 2261 (1996).

${ }^{10}$ L. Demirel and S. Granick, J. Chem. Phys. 109, 6889 (1998).

${ }^{11}$ J. Klein and E. Kumacheva, Science 269, 816 (1995).

${ }^{12}$ E. Kumacheva and J. Klein, J. Chem. Phys. 108, 7010 (1998).
${ }^{13}$ B. N. J. Persson and E. Tosatti, Phys. Rev. B 50, 5590 (1994).

${ }^{14}$ N. J. Persson, Chem. Phys. Lett. 324, 231 (2000). See also Fractals and Disordered Systems, edited by A. Bunde and S. Havlin (Springer, Berlin, 1991); J. S. Langer, Phys. Rev. A 36, 3350 (1987); E. Brener, Phys. Rev. Lett. 71, 3653 (1993).

${ }^{15} \mathrm{U}$. Raviv and J. Klein, private communications.

${ }^{16}$ A. Dhinojwala and S. Granick, J. Am. Chem. Soc. 119, 241 (1997).

${ }^{17}$ F. Mugele and M. Salmeron, Phys. Rev. Lett. 84, 5796 (2000).

${ }^{18}$ F. Mugele and M. Salmeron, J. Chem. Phys. 114, 1831 (2001).

${ }^{19}$ F. Mugele, S. Baldelli, G. A. Somorjai, and M. Salmeron, J. Phys. Chem. B 104, 3140 (2000).

${ }^{20}$ B. N. J. Persson and P. Ballone, Solid State Commun. 115, 599 (2000).

${ }^{21}$ B. N. J. Persson and P. Ballone, J. Chem. Phys. 112, 9524 (2000).

${ }^{22}$ It should be noted that the structure in Fig. 1 is made somewhat complicated by another effect - the increase of the contact area with time due to adhesion. Thus the black circular ridge at the bottom right of the contact area results not from squeezing fluid out but from the change in transmittance properties in this adhesion region.

${ }^{23}$ J. G. Dash, Films on Solid Surfaces (Academic, New York, 1975).

${ }^{24}$ L. D. Landau and E. M. Lifshitz, Theory of Elasticity (Pergamon, Berlin, 1986).

${ }^{25}$ See, e.g., Fractals and Disordered Systems, edited by A. Bunde and S. Havlin (Springer, New York, 1991).

${ }^{26}$ D. Kessler, J. Koplik, and H. Levine, Adv. Phys. 37, 255 (1988).

${ }^{27} \mathrm{P}$. Wesseling, An Introduction to Multigrid Methods (Wiley, New York, 1992).

${ }^{28}$ Numerical Recipes in C: The Art of Scientific Computing (Cambridge University Press, Cambridge, 1988-1992).

${ }^{29}$ J. A. Sethian, Level Set Methods and Fast Marching Methods (Cambridge University Press, Cambridge, 1999). 